\title{
A caracterização de uma base orientadora para ensinar o conteúdo de mamíferos: contribuições da Teoria de P. Ya. Galperin
}

\author{
Alessandro Augusto de Barros Façanha (D), Micarla Silva de Azevedo (i) \& Nathany \\ Morais de Souza
}

Universidade Federal do Rio Grande do Norte, Centro Regional de Ensino Superior do Seridó, Departamento de Educação, Joaquim Gregório, Penedo 59300-000, Caicó, Rio Grande do Norte, Brasil. Email: abfacanha@gmail.com, silvamicarla14@gmail.com, nathany.morais@hotmail.com

Façanha A.A.B., Azevedo M.S. \& Souza N.M. (2020) A caracterização de uma base orientadora para ensinar o conteúdo de mamíferos: contribuições da Teoria de P. Ya. Galperin. Pesquisa e Ensino em Ciências Exatas e da Natureza, 4: e1546. http://dx.doi.org/10.29215/pecen.v4i0.1546

Editor acadêmico: Carlos Alberto de Oliveira Magalhães Júnior. Recebido: 10 julho 2020. Aceito: 06 outubro 2020. Publicado: 09 novembro 2020.

Resumo: Este estudo traz uma contribuição para o ensino de ciências e objetiva investigar uma base orientadora para o conteúdo de mamíferos. Metodologicamente se fundamenta na elaboração do esquema da base orientadora completa para o estudo dos mamíferos em enfoque sistêmico da Atividade e nos pressupostos da invariante da ação para assim caracterizar e elaborar um esquema da base orientadora completa da ação, o EBOCA para se identificar o conceito de mamíferos, a partir do qual se problematizam as questões de ensino sob a perspectiva da Teoria da Atividade. Como resultado se propõe esse modelo orientador como uma referência didático-metodológica para o ensino das ciências em função de se possibilitar um processo de aprendizagem pautado nos indicadores qualitativos da ação, cuja vantagem reside no fato de uma atividade consciente e com alto grau de transferência na resolução de problemas.

Palavras chave: Conceitos científicos, ensino de mamíferos, orientação da ação.

The characterization of a guiding activity for teaching the mammalian content: contributions to the Theory of P. Ya. Galperin

Abstract: This study makes a contribution to science education and aims to investigate a guiding basis for mammalian content. Methodologically it is based on the elaboration of the scheme of the complete guiding base for the study of mammals in a systemic focus of the Activity and on the assumptions of the action invariant to characterize and elaborate a scheme of the complete guiding base of the action, the EBOCA to identify the concept of mammals, from which the issues of teaching are problematized from the perspective of Activity Theory. As a result, this guiding model is proposed as a didactic-methodological reference for the teaching of sciences in order to enable a learning process based on the qualitative indicators of action, whose advantage lies in the fact of a conscious activity and with a high degree of transference in problem solving.

Key words: Scientific concepts, teaching of mammals, guiding activity.

\section{Introdução}

O ensino dos conceitos, por fazer parte da formação e do desenvolvimento cognitivo do indivíduo, está presente nas teorias psicológicas em geral. No âmbito do enfoque históricocultural, remonta aos estudos de Vygotski (1993), aonde essa temática se encontra presente a 
partir da defesa de que é na escola que ocorre o processo formal de desenvolvimento psíquico, e a aquisição de conceitos científicos como um dos princípios do processo de ensino-aprendizagem.

Na perspectiva Vygotskiana, a formação dos conceitos científicos ou escolares, resulta de transformações qualitativas do pensamento dos indivíduos, as quais mediadas pelo aspecto social dos signos e da linguagem, estabelece a aprendizagem como consequência da atividade. Visto isso, o presente estudo objetiva investigar uma base orientadora para o conteúdo de mamíferos, que considere as invariantes desse conceito.

O caráter social da aprendizagem se reflete por meio da internalização, que constitui o aspecto intrapsíquico da atividade e acarreta no intercâmbio cultural entre os sujeitos, os quais através da atividade colaborativa interpsíquica, desenvolvem os aspectos subjetivos desse processo, que no caso específico dos conceitos, possibilita a aquisição de sentidos em torno dos significados presentes nos conteúdos escolares (Tacca \& Gonzalez Rey 2008).

Nesse contexto, a aprendizagem dos conceitos resulta na concepção de que o desenvolvimento do sujeito no ambiente escolar é uma consequência da obutchénie ${ }^{1}$, a qual estabelece, segundo os ideais vygotskianos, um marco revolucionário da psicologia históricocultural no seu âmbito pedagógico, pois, interpreta o desenvolvimento intelectual dos indivíduos em função da formação de habilidades e ações mentais ${ }^{1}$ Refere-se a uma concepção-ação não cultural
para nós, mas em que ambas dimensões e
processos encontram-se em unidade. Na
perspectiva histórico-cultural essa unidade está
na relação com o desenvolvimento, uma vez que
a finalidade e o objeto da obutchénie (ensino-
aprendizagem) é o desenvolvimento; portanto,
refere-se ao processo ensino-aprendizagem-
desenvolvimento (Longarezi \& Puentes 2017: 12). relacionadas ao desenvolvimento do pensamento teórico.

Conceber a ideia que o conhecimento da essência de um fenômeno, em termos de suas características invariantes, é o que determina a assimilação do caráter universalizante do objeto da aprendizagem, assim, como explica Davidov \& Shuare (1988: 311), "conhecer a essência significa encontrar o geral como base, uma fonte da variedade de fenômenos e suas relações na existência do concreto".

Consequentemente, a assimilação dos conhecimentos e formação dos conceitos científicos, requer dos indivíduos a internalização das características essenciais e necessárias do objeto da aprendizagem em questão. Como designa Nuñez (2009), consiste na apropriação de casos particulares em função de seu aspecto generalizante, a fim de possibilitar uma ampla transferência de conhecimentos para a resolução de uma classe de problemas dentro do limite de aplicação desse conceito. É nesse contexto que se defende o ensino de conceitos científicos baseado na concepção da orientação da ação de P. Ya. Galperin.

A base orientadora (BOA) representa, do ponto de vista cognitivo, as condições necessárias para o planejamento, execução racional e autorregulação da atividade. Ao se caracterizar um objeto de aprendizagem (ação ou conceito), em função de sua estrutura invariante, se possibilita uma assimilação em torno do conhecimento conceitual e operativo, essencial para a representação mental deste objeto de aprendizagem.

Interpretando esse processo com base no que escreveu Galperin (1986), consiste em compreender que, reside na orientação a estrutura generalizante de uma ação que, em termos cognoscitivos, permite ao sujeito antecipar, planejar e executar uma atividade com caráter consciente e autorregulado, implicando, portanto, em um processo de aprendizado estável do ponto de vista da assimilação dos conceitos.

Nesse sentido, se elabora este estudo com o propósito de responder a seguinte questão: com base na Teoria da Formação Planejada das Ações Mentais e dos Conceitos de P. Ya. Galperin, é possível se elaborar uma base orientadora que caracterize a invariante do conteúdo de mamíferos para ser utilizado no Ensino Fundamental durante as aulas de ciências?

Essa questão central se alia à problemática enfrentada no âmbito da educação científica, em especial, ao ensino dos conceitos, pois, durante essa etapa da Educação Básica, se percebe que há uma necessidade, quer no âmbito da formação dos professores, quer no âmbito da didática em sala de aula de se executarem estratégias de ensino mais alinhadas ao processo de alfabetização científica. Assim, tem como fim garantir uma aprendizagem compatível com o que 
preconiza as matrizes de referência avaliativas acerca dos conceitos científicos e de sua relação com a formação do pensamento teórico (OECD 2013; INEP 2015).

Por conseguinte, esse estudo se fundamenta nos conceitos de orientação da ação em função da elaboração de um esquema orientador que contemple o caráter invariante do conceito de mamíferos, bem como o marco teórico da psicologia histórico-cultural da aprendizagem.

\section{Aspectos conceituais da Teoria de Galperin: um enfoque para a orientação da ação no ensino dos conceitos}

P. Ya. Galperin (1902-1988) foi um psicólogo da Escola Soviética do psiquismo que dedicou seus estudos à assimilação dos conceitos e formação das ações mentais, em decorrência da teoria da atividade e dos estudos da internalização de L. S. Vygotski.

Tinha como foco compreender o processo de sistematização envolvido na formação das ações mentais decorrentes do desenvolvimento cognoscitivo em função do que, estabeleceu as bases da Teoria da Formação Planejada das Ações Mentais e dos Conceitos (TFPAMC). De acordo com essa teoria, o processo de assimilação e consequente formação das ações mentais decorre em função de etapas planejadas, que se configuram em uma orientação, execução e controle das ações, as quais passam por uma etapa materializada, pela linguagem e, por conseguinte, se representam mentalmente (Galperin 1986).

A orientação da ação, conforme expressa Nuñez \& Ramalho (2013: 12) "é o modelo da atividade, porque reflete todas as partes estruturais e funcionais da mesma. Nela se inclui o sistema de condições, as quais se apoia o aluno para resolver as tarefas do mesmo tipo, como resultado da investigação-orientação. Assim, a orientação da ação é o objeto da psicologia que materializa a estrutura das ações em seu processo de intercâmbio entre a realidade externa e as ações internalizadas pela psique.

Todavia, apesar da orientação se configurar como a estrutura para a formação das ações mentais e também como uma categoria central da aprendizagem, não é toda base orientadora que reflete a melhor representação em termos do seu conteúdo conceitual e operativo de uma ação. Em sua teoria, Galperin (1986) estabelece quatro tipos de orientação de acordo com critérios de qualidade de uma ação e sua regulação para a aprendizagem.

Nesta condição é possível observar em detalhes a caracterização de orientação quanto a sua tipologia, conforme o Quadro 1.

Quadro 1. Caracterização dos tipos de orientação de acordo com os critérios da TFPAMC. Fonte: Galperin (1986: adaptado pelos autores).

\begin{tabular}{|c|c|}
\hline Tipo de Orientação & Descrição \\
\hline BOA do tipo I & $\begin{array}{l}\text { A orientação se refere a um caso em particular e baseada na aprendizagem por } \\
\text { tentativa/erro e cujo aspecto pedagógico presente consiste na repetição da ação. }\end{array}$ \\
\hline BOA do tipo II & $\begin{array}{l}\text { Caracteriza-se pela existência das condições necessárias para a execução da ação, } \\
\text { entretanto, consiste em uma orientação exclusivamente diretiva, de modo que } \\
\text { não possibilita que o sujeito elabore suas próprias ações a partir da base de modo } \\
\text { que não potencializa o aprendizado pelo erro e limita o aprender à transferência } \\
\text { das operações. }\end{array}$ \\
\hline BOA do tipo III & $\begin{array}{l}\text { É denominada de orientação de composição completa, pois as orientações } \\
\text { presentes se referem a uma classe de fenômenos conferindo à base um caráter } \\
\text { mais generalista, dessa forma, confere ao sujeito a capacidade de elaboração e } \\
\text { inferência a partir da orientação. Consiste em uma proposta mais ampla de } \\
\text { aprendizado e execução da ação. }\end{array}$ \\
\hline BOA do tipo IV & $\begin{array}{l}\text { Diferencia-se da anterior apenas pelo fato de ser dada ao aluno, assim também se } \\
\text { referência pelos mesmos pontos didático-metodológicos em relação à classe de } \\
\text { fenômenos e de generalização. }\end{array}$ \\
\hline
\end{tabular}

Em termos da aprendizagem, a base orientadora do tipo III é a que representa em sua composição o caráter mais generalizado da ação, aplicando-se, portanto, a um conjunto de 
fenômenos e tarefas de uma determinada classe. De acordo com as explicações de Nuñez (2009), corresponde a orientação mais adequada em relação ao caráter invariante da ação ou do objeto da aprendizagem, pois, como representa a orientação subjetiva do estudante exerce um papel importante na transferência de conhecimentos e nível de consciência para a resolução de problemas em relação ao seu aspecto invariante.

\footnotetext{
A invariante se refere à essência que justifica um conjunto de situaçõesproblemas e os procedimentos gerais de sua solução: invariante conceitual e invariante procedimental. A invariante se relaciona com o conjunto de situações segundo o par de categorias dialéticas: essência-fenômeno. Essas invariantes constituem o conteúdo da BOA tipo III (Nuñez 2009: 103).
}

Consequentemente, em relação ao aprendizado dos conceitos científicos, essa representação corresponde ao conhecimento desejado e esperado para os estudantes após o processo formal de educação escolar, pois, caso essa orientação esteja representada mentalmente sob a forma da base orientadora dos estudantes, significa que os mesmos assimilaram conceitualmente um objeto ou fenômeno, em função de seu caráter invariante.

Entretanto, tomando como premissa os dados evidenciados no cenário de proficiência em ciências, há de se considerar que a orientação dos estudantes acerca dos conceitos científicos ainda se encontra longe dessa realidade (OECD 2013). Nesse aspecto, é possível correlacionar essas dificuldades de aprendizagem a uma orientação inadequada para promoção da assimilação dos conceitos.

Conforme se estabelece pela TFPAMC, um dos papéis do professor é o de possibilitar, através da didática, situações e estratégias de aprendizagem que se configurem, no âmbito da orientação, como um processo de negociação entre a base orientadora dos estudantes e um modelo de referência de orientação que represente o caráter invariante do objeto a ser ensinado.

Essa premissa é o que Galperin (1982) define como sendo a caracterização de um esquema da base orientadora completa da ação (EBOCA), que nada mais é do que a representação da base orientadora de referência para o aprendizado consciente, generalizado e de amplo grau de transferência dentro de seu limite de aplicação.

A elaboração do EBOCA, portanto, coincide no campo da didática e do ensino como um aspecto inerente à profissionalidade docente no sentido de garantir um esquema de referência para ensinar um determinado conceito ou habilidade, através do qual é possível modificar a compreensão dos estudantes a partir da reestruturação de sua base orientadora, em função de se conceber a orientação como uma categoria relacionada à aprendizagem.

\section{O EBOCA e a aprendizagem de conceitos}

Como descrito anteriormente, a aprendizagem no âmbito da psicologia pedagógica soviética se constitui como um processo psíquico de internalização das ações externas por meio da mediatização sociocultural (Talizina 2000). Essa concepção, como explica Galperin (1986), contribui para uma reconceituação do processo de aprendizagem, como um contraponto ao modelo condutista sobre o comportamento e acerca do padrão cognitivista associado à adaptação e racionalismo técnico.

No campo dos conceitos, concebe à atividade uma via para a formação das funções psíquicas e, consequentemente, para a formação das ações mentais, que não apenas amplia a concepção sobre a aprendizagem, como promove um entendimento sobre o papel da consciência no âmbito do desenvolvimento dos sujeitos, pois possibilita sistematizar, tanto epistemologicamente como metodologicamente, uma explicação para a assimilação.

Nesse sentido, o conceito da orientação da ação, como estrutura diretiva da aprendizagem ocorre em função do fato da $\mathrm{BOA}$ representar as condições para o planejamento e execução racional de uma ação, além de um sistema autorregulado sobre um objeto de aprendizagem. Resulta, portanto, como um produto internalizado da atividade. Como ressaltam Nuñez \& Ramalho (2018: 18): 
A aprendizagem, como processo de assimilação dos conteúdos, avança do geral para o concreto, por meio de atividade conjunta e por mecanismos de simbolização do conteúdo, usando a linguagem como meio de formulação linguística de relações e de conscientização do estudante. (...) o processo de internalização da atividade externa em interna é concebido como um ciclo cognoscitivo, no qual se destacam momentos funcionais que, mesmo não sendo considerados de forma linear, podem ser separados metodologicamente para a análise didática.

No entanto, como a orientação da ação é uma representação subjetiva e internalizada em função das experiências formativas dos sujeitos, no âmbito da TFPAMC, se estabeleceu que a orientação de referência para o aprendizado deveria consistir da caracterização da estrutura invariante da ação ou do objeto a ser assimilado, que, no caso específico dos conceitos científicos, seria um dos aspectos promotores do pensamento teórico nos estudantes.

Deriva daí, portanto, o conceito de esquema da base orientadora completa da ação, o EBOCA, que segundo Galperin (1982), define, a partir de um limite de generalização, as características essenciais da atividade cognoscitiva.

No âmbito do processo de ensino-aprendizagem, como explica Nuñez et al. (2018), o EBOCA representa uma referência para o professor, pois contém exatamente as condições essenciais para a adequada execução da ação e do controle, que no contexto do ensino de conceitos pode ser entendido como a caracterização de um esquema de orientação externa que serve de balizador para a negociação entre a BOA dos estudantes e o conhecimento planejado pelo professor como objetivo de aprendizado. Portanto:

\footnotetext{
O EBOCA fornece aos estudantes uma ferramenta cultural para a generalização teórica, que permite a compreensão de um conjunto de situações ou de um dado domínio do conhecimento que define seus limites de aplicação ou o grau de generalização. Essa é uma condição essencial para a formação de ações mentais e dos conceitos com alta possibilidade de transferência às novas situaçõesproblema. Na base do conteúdo do EBOCA, o estudante pode refletir sobre o que já sabe, sobre o que é capaz de fazer e sobre o que ainda deve aprender e, assim, planejar essa aprendizagem (Nuñez \& Ramalho 2018: 8-9).
}

Dessa maneira, a caracterização do EBOCA no campo da didática em sala de aula, representa o modelo orientador elaborado pelo professor, a partir do qual se planejam as atividades docentes no intuito de promover uma negociação entre a orientação dos estudantes e esse modelo de referência, segundo o qual, as tarefas e atividades colaborativas devem estar alinhadas.

Isso possibilita, dado o nível de compreensão sistematizada sobre uma ação ou conceito, tornar o processo de ensino-aprendizagem, uma ação racional que permite os estudantes tomarem consciência do que estão executando e porque estão executando, consequentemente, no caso da aprendizagem de conceitos nas ciências, torna possível estabelecer a compreensão dos fenômenos em nível explicativo e não meramente descritivo.

Alinha-se no aspecto da ação racional, as prerrogativas da alfabetização científica preconizada pelas matrizes de referência que avaliam a proficiência científica, pois de acordo com esses pressupostos, os indivíduos precisam agir, em função da assimilação de conceitos, em torno da resolução de problemas e tomada de decisão (OCDE 2015).

Nesse sentido, o EBOCA se associa a essas demandas da formação do pensamento teórico que permitem aos estudantes a assimilação de um conceito em função de seu caráter essencial, afinal, de acordo com a TFPAMC, o processo de assimilação consiste na formação por etapas das ações e seu aperfeiçoamento, no sentido de se configurar como a transformação da forma material-objetal em mental-objetal, que redundam na automatização das ações e formação de hábitos, os quais em termos da aprendizagem se configuram como uma transformação da base orientadora (Galperin et al. 1987).

Por conseguinte, a elaboração de um EBOCA como referência para o ensino de conceitos, se constitui em termos didáticos e pedagógicos, como um requisito de sua 
profissionalidade uma vez que, possibilita uma ação docente voltada, em termos cognitivos, para o desenvolvimento de ações mentais que representem uma concepção de aprendizagem mais estável.

No escopo desse estudo, a caracterização de um modelo de referência acerca do conceito de mamíferos para ser aplicado no Ensino Fundamental, pode propiciar uma aprendizagem mais efetiva do ponto de vista do seu grau de transferência para diversas situações-problema, bem como evitando uma compreensão particularizada do conceito por parte dos estudantes que, via de regra, se aplica ao modelo reprodutivista empregado pelo ensino tradicional vigente em grande parte das escolas.

A próxima seçcão traz essa caracterização a partir da necessidade de se ensinar esse conteúdo como parte de um processo de compreensão dos conceitos associados às ciências.

\section{A orientação acerca do conceito de mamíferos no âmbito da Educação Básica}

Tomando como referência as atuais diretrizes que norteiam o ensino das ciências na Educação Básica brasileira, se pode perceber que há, tanto em relação aos Parâmetros Curriculares Nacionais (PCN) como nas atualizações trazidas pela Base Nacional Comum Curricular (BNCC), uma concepção de aprendizagem em função da formação de habilidades e conceitos com o propósito do desenvolvimento do pensamento para a resolução de problemas e atuação na sociedade.

Na BNCC, competência é definida como a mobilização de conhecimentos (conceitos e procedimentos), habilidades (práticas, cognitivas e socioemocionais), atitudes e valores para resolver demandas complexas da vida cotidiana, do pleno exercício da cidadania e do mundo do trabalho (BRASIL 2017: 8, grifo nosso).

Nessa perspectiva, em função da TFPAMC, torna-se imprescindível, do ponto de vista da aprendizagem, reconhecer a orientação acerca de habilidades e conceitos representada nestas diretrizes, pois, a partir dessa referência o professor pode planejar sua atividade docente em função da restruturação da base orientadora como estratégia de ensino e aprendizagem. Nesse caso específico, é preciso entender os normativos e diretrizes como balizadores da orientação, pois estabelecem a referência para a atividade escolar.

A BNCC, por exemplo, direciona conteúdos que irão fazer parte do currículo de formação dos estudantes através de unidades temáticas. Em relação ao conteúdo dos mamíferos, o qual faz parte dos conteúdos curriculares do terceiro ano do Ensino Fundamental, se estrutura em função de secções temáticas das quais se destaca a orientação para o ensino no contexto da vida e evolução, a partir da qual se apoiam os objetivos de aprendizagem em torno das características e desenvolvimento dos animais.

Nesse aspecto, se orientam uma série de habilidades, isto é conhecimentos conceituais e operativos, a partir dos quais os estudantes precisam ter habilidades do pensamento lógico para "comparar alguns animais e organizar grupos com base em características externas comuns (presença de penas, pelos, escamas, bico, garras, antenas, patas etc)” (BRASIL 2017: 336-337).

Como consequência, a BNCG apresenta os conteúdos referentes ao conceito de mamíferos em função da caracterização de seu modo de vida (alimentação, reprodução, locomoção), além da identificação de peculiaridades como o desenvolvimento e as diferenças com as demais classes de animais em função dessas categorias.

Comparando essas orientações e a concepção explicada anteriormente acerca do caráter invariante e generalizado que deve estar associado à base orientadora para o desenvolvimento do pensamento teórico, se percebe que, apesar de associado à habilidades do pensamento lógico, como comparação e identificação, por exemplo, em relação ao enfoque histórico-cultural e da TFPAMC, há uma tendência à particularização do conceito em função de situações-problema específicas. 
Correlacionando essas ideias ao que é trazido pelos livros didáticos, os quais, em termos da orientação representam uma BOA do tipo II, no aspecto de assegurar a transferência de aprendizagem para situações de aplicação dos conceitos, é possível se interpretar essas ideias a partir do que expressa Rosa (2017), pois, segundo esses autores, as estratégias de ensino presentes no material didático recorrem de maneira excessiva à observação e ao modelo reprodutivo de fixação do conteúdo sob a forma de atividades restritas e muitas vezes superficiais dos conceitos.

Assim, a partir de uma interpretação à luz da Teoria da Atividade e dos aspectos didáticos do enfoque histórico-cultural, sobretudo em relação à TFPAMC, é preciso destacar que, em relação à base orientadora, há uma exacerbação do conhecimento observacional, o que limita o conhecimento conceitual e operativo dos estudantes, pois não possibilita uma dimensão compreensiva em torno da invariante do conceito.

Por esses aspectos é que se defende a ideia, em consonância com os autores da didática desenvolvimental, que é preciso reconsiderar o modelo orientador trazido pelos referenciais curriculares e configurados muitas vezes nos materiais didáticos vigentes, pois, além de representarem uma concepção condutista de aprendizagem, contribuem pouco no aspecto da formação do pensamento teórico em relação aos conceitos, pois estão pautados basicamente na reprodução da orientação do livro didático.

Como contraponto, aprofundar a caracterização da estrutura invariante em torno dos conceitos, possibilita uma maior plenitude na assimilação e maior nível de compreensão em função do caráter consciente envolvido. Afinal, como explicam Puentes \& Longarezi (2017: 208), em relação à aprendizagem:

\footnotetext{
[...] Resultam naqueles que conhecimentos que constituem a base de muitos conteúdos das matérias escolares, diferentemente dos conhecimentos particulares que a escola tradicional se esforça em ensinar. Por serem invariantes, esses conteúdos permitem a aquisição de conceitos científicos e ações mentais que podem ser transferidos para novas situações da mesma espécie pelo alto nível de abstração e generalização em que se formam.
}

A secção a seguir descreve em termos metodológicos o percurso e a fundamentação para a elaboração e caracterização de uma orientação de referência para o ensino do conceito de mamíferos no Ensino Fundamental evidenciando assim, uma proposta de estudo que integra essas nuances citadas como uma estratégia de formação dos estudantes.

\section{Metodologia}

Esse estudo se fundamenta em torno de uma perspectiva histórico-cultural da Atividade relacionada ao ensino de conceitos como estratégia relacionada à orientação da ação. Implica na defesa de que o desenvolvimento cognitivo dos sujeitos precisa estar amparado em um esquema orientador capaz de representar os conceitos científicos em função de seu caráter sistêmico, ou seja, a partir de sua caracterização funcional-estrutural (Talízina 2000).

Se enquadra no campo das pesquisas aplicadas ao ensino das ciências, a partir da qual se objetiva problematizar o aspecto da orientação da ação relacionada a formação de conceitos científicos com a proposição de que a partir da elaboração de uma base orientadora caracterizada em função das invariantes estruturais e funcionais relativas ao conceito de mamíferos é possível se estruturar uma proposta didática mais efetiva do ponto de vista da cognoscitivo com maior potencial de transferência de aprendizagem como pressupõem os estudos pautados na Teoria da Formação Planejada das Ações Mentais e dos Conceitos de P. Ya. Galperin.

Com esse propósito, metodologicamente, o estudo se baseia no chamado método sistêmico da atividade, a partir do qual, se compreende que um sistema de ensino eficiente precisa se fundamentar em torno da caracterização das propriedades funcionais e estruturais de um conceito ou conteúdo para instrução didática (Salmina 1988). 
Assim, em consonância com os estudos aplicados à formação das habilidades cognitivas proposto por Nuñez (2009), o objeto de estudo aqui definido, ou seja, a base orientadora relacionada ao conceito de mamíferos, passa a ser compreendida como uma caracterização funcional e estrutural da ação de identificação dessa classe de seres vivos, em função de suas propriedades essenciais para a assimilação.

Consequentemente, se apresenta como objeto de investigação metodológica e também como resultado do estudo, a elaboração de uma orientação de referência para aplicação do ensino de ciências em resposta ao objetivo proposto por esse estudo, isto é, a elaboração de uma base orientadora que caracterize a invariante do conteúdo de mamíferos para ser utilizado no Ensino Fundamental durante as aulas de ciências.

Tal proposição adquire suporte metodológico de pesquisa quando se consideram os pressupostos do Método Teórico da Atividade, através do qual, de acordo com as explicações de Talízina (1987), se realiza o mapeamento da atividade em função de sua invariante operacional e de um núcleo conceitual do objeto a ser assimilado.

Nessa lógica, a fim de se caracterizar os aspectos invariantes relacionados ao conceito se realizou o delineamento do mapa da atividade que de acordo com Galperin (1986), representa as propriedades intrínsecas do objeto a ser assimilado. Tal mapeamento foi realizado tendo como base o levantamento de fontes válidas do conhecimento das ciências, tendo como critério as seguintes características: representar uma fonte de informação validada pela comunidade científica, configurar como informação produzida por meios acadêmicos ou fazer parte de material didático para o ensino de ciências chancelado por órgão de educação ou editoração educacional.

Nesse sentido, a estrutura do mapa da atividade relativo à caracterização de um modelo de orientação para o ensino conceitual de mamíferos seguiu o seguinte desenho metodológico de acordo com a fonte e dos critérios de validade da informação (Quadro 2):

Quadro 2. Fontes utilizadas para a caracterização da invariante relacionada ao conceito de mamíferos e critério metodológico de escolha.

\begin{tabular}{|c|c|}
\hline Fonte & $\begin{array}{l}\text { Critério para compor fonte de pesquisa do mapa da } \\
\text { atividade }\end{array}$ \\
\hline $\begin{array}{l}\text { Livro didático Ed. FTD - Gowdak D. \& Martins E. (2002) } \\
\text { Ciências: novo pensar. São Paulo: FTD. } 392 \text { p. }\end{array}$ & $\begin{array}{l}\text { Material referendado pelo Plano Nacional do Livro } \\
\text { Didático (PNLD) com critério de validação do } \\
\text { Ministério da Educação (MEC). }\end{array}$ \\
\hline $\begin{array}{l}\text { Livro didático Ed. Moderna - Yamamoto A.C.A. (2017) } \\
\text { Buriti mais: ciências. São Paulo: Moderna. } 32 \text { p. }\end{array}$ & $\begin{array}{l}\text { Material referendado pelo Plano Nacional do Livro } \\
\text { Didático (PNLD) com critério de validação do } \\
\text { Ministério da Educação (MEC). }\end{array}$ \\
\hline Revista Galileu de divulgação científica & $\begin{array}{l}\text { Sítio de informação vinculada a divulgação das } \\
\text { ciências com corpo editorial reconhecido. }\end{array}$ \\
\hline $\begin{array}{l}\text { Grupo de pesquisas em Biodiversidade de mamíferos - } \\
\text { UNICENTRO. http://dgp.cnpq.br/dgp/espelhogrupo/39288 }\end{array}$ & $\begin{array}{l}\text { Grupo de pesquisa cadastrado na base do } \mathrm{CNPq} \text { com } \\
\text { produção validada pela comunidade acadêmica } \\
\text { reconhecida na área do estudo }\end{array}$ \\
\hline $\begin{array}{l}\text { Pesquisas do grupo de ecologia de Mamíferos - UFU. } \\
\text { http://dgp.cnpq.br/dgp/espelhogrupo/19306 }\end{array}$ & $\begin{array}{l}\text { Grupo de pesquisa cadastrado na base do } \mathrm{CNPq} \text { com } \\
\text { produção validada pela comunidade acadêmica } \\
\text { reconhecida na área do estudo }\end{array}$ \\
\hline
\end{tabular}

Essa estratégia possibilita caracterizar o conceito em função do que se denomina de modelo do objeto (conhecimento conceitual) e modelo da ação (conhecimento operativo), que de acordo com a TFPAMC, em relação ao planejamento do professor, possibilita referendar um esquema completo da orientação da ação, o qual, em função do que foi descrito na fundamentação teórica desse estudo, se configura como o EBOCA para o ensino de um conceito científico.

Desse modo será apresentado nos resultados dessa pesquisa como parte do referencial didático-formativo para o ensino do conceito de mamíferos em aulas de ciências de modo a possibilitar o aprendizado para além do aspecto da memorização ou da particularização do mesmo, em casos específicos que envolvem o conteúdo. 
Desta forma, em termos metodológicos, essa estratégia adotada permitiu, como explica Galperin (2001), representar o mapa da atividade para a ação de conceituar mamíferos, ao mesmo tempo que elucidou a compreensão sobre as operações necessárias para se assimilar o conceito em sua extensão generalizada, o que, em termos do processo de ensino, permite estruturar uma referência fora dos parâmetros de exceção e particularidades, às vezes, impostas pelo modelo da orientação do livro didático.

Nesse aspecto, resulta como produto dessa investigação a elaboração de uma orientação de referência para o ensino de ciências nos anos iniciais do Ensino Fundamental de forma a possibilitar um processo de aprendizagem mais estável e duradouro, pois se associa diretamente ao planejamento, execução racional e autorregulação pelo estudante. A seç̧ão, propõe a caracterização desse modelo orientador como uma proposta para o ensino do conteúdo de mamíferos e suas discussões em torno da didática e do processo de ensino-aprendizagem.

\section{Resultados e Discussão}

Em consonância com o objetivo central dessa investigação, o primeiro aspecto a ser apresentado e discutido enquanto resultado diz respeito ao mapeamento das invariantes estruturais e funcionais relacionadas ao conceito de mamíferos, os quais foram obtidos em resposta ao método teórico da atividade a fim de se elaborar posteriormente o esquema orientador de referência.

Nesse sentido, tomando a estrutura relativa ao mapa da atividade para identificar mamíferos, se tem a seguinte caracterização da orientação trazida em cada uma das fontes investigadas, conforme se descreve no Quadro 3, a seguir:

Quadro 3. Caracterização do modelo de orientação relativo ao conceito de mamíferos presente nas fontes utilizadas para estabelecer o caráter invariante da ação.

\begin{tabular}{|c|c|c|}
\hline Fonte & \multicolumn{2}{|c|}{$\begin{array}{l}\text { Caráter estrutural-funcional presente no modelo de orientação acerca } \\
\text { do conteúdo para identificar mamíferos }\end{array}$} \\
\hline & Modelo do objeto & Modelo da ação \\
\hline $\begin{array}{l}\text { Livro didático Ed. FTD - Gowdak D. \& } \\
\text { Martins E. (2002) Ciências: Novo Pensar. } \\
\text { São Paulo: FTD. } 392 \text { p. }\end{array}$ & $\begin{array}{l}\text { Animais de habitat aquático ou } \\
\text { terrestre, vivíparos. Maioria tem } \\
\text { corpo coberto por pelos, filhotes } \\
\text { mamam. }\end{array}$ & $\begin{array}{l}\text { Não apresenta orientação em } \\
\text { relação ao conhecimento } \\
\text { operativo ou procedimental. }\end{array}$ \\
\hline $\begin{array}{l}\text { Livro didático Ed. Moderna - Yamamoto } \\
\text { A.C.A. (2017) Buriti mais: ciências. São } \\
\text { Paulo: Moderna. } 32 \text { p. }\end{array}$ & $\begin{array}{l}\text { Animais com corpo coberto de } \\
\text { pelos. Seus filhotes se alimentam } \\
\text { do leite produzido pela fêmea. }\end{array}$ & $\begin{array}{l}\text { Não apresenta orientação em } \\
\text { relação ao conhecimento } \\
\text { operativo ou procedimental. }\end{array}$ \\
\hline Revista Galileu de divulgação científica. & $\begin{array}{l}\text { Corpo majoritariamente coberto } \\
\text { por pelos, animais de circulação } \\
\text { fechada. }\end{array}$ & $\begin{array}{l}\text { Não apresenta conhecimento } \\
\text { operativo. }\end{array}$ \\
\hline $\begin{array}{l}\text { Grupo de pesquisas em Biodiversidade de } \\
\text { mamíferos - UNICENTRO. http://gpp.cnp } \\
\text { q.br/dgp/espelhogrupo/39288 }\end{array}$ & $\begin{array}{l}\text { Animais de fecundação interna, } \\
\text { multiplicidade de desenvolvimento } \\
\text { caráter placentário nos terrestres, } \\
\text { diafragmáticos, circulação fechada } \\
\text { e completa. }\end{array}$ & $\begin{array}{l}\text { Identificação da regulação } \\
\text { corpórea, presença de glândulas } \\
\text { mamárias e regulação hormonal. }\end{array}$ \\
\hline $\begin{array}{l}\text { Pesquisas do grupo de ecologia de } \\
\text { Mamíferos - UFU. http://dgp.cnpq.br/dgp/ } \\
\text { espelhogrupo/19306 }\end{array}$ & $\begin{array}{l}\text { Seres de ampla diversidade com } \\
\text { desenvolvimento } \\
\text { heterótrofos, circulação dupla e } \\
\text { fechada. }\end{array}$ & $\begin{array}{l}\text { Comparação adaptativa, aspecto } \\
\text { nutricional com produção de } \\
\text { alimento por indução hormonal } \\
\text { pós-gestação. }\end{array}$ \\
\hline
\end{tabular}

O mapeamento da invariante possibilitou constatar as características generalizantes acerca do conceito de mamíferos, as quais, tomando como referência para o ensino das ciências, possibilita uma maior eficiência em termos da aprendizagem se comparada à base orientadora representada pelos livros didáticos tradicionais. Pois, devido ao caráter particularizado expresso pela orientação presente nesses materiais, é possível correlacionar algumas das dificuldades dos estudantes em relação à essa perspectiva, sobretudo quando os mesmos se deparam com situações diferentes das discutidas em sala ou pelos textos dos livros. 
No caso dos mamíferos, conforme declaram Oliveira et al. (2013), um erro comum reside em torno da orientação não generalizada como as observadas em sala de aula, quando, por exemplo, o aspecto particularizado referente à presença de pelos, que se manifesta no senso comum de alguns indivíduos, se apresenta como uma dificuldade de aprendizagem de estudantes, pois acabam generalizando uma característica que é particular.

Essas impressões são observadas por exemplo, nas análises do livro didático presente nos estudos de Silva et al. (2012) e Oliveira et al. (2013), os quais descrevem que ainda há algumas imprecisões muito comuns quando se analisa o conteúdo de mamíferos presentes nos materiais didáticos, como por exemplo, imprecisões relativas à exemplificação das espécies onde é bastante comum se desconsiderar o aspecto regional dos estudantes ao abordar a temática dos mamíferos, assim, se incorrendo em possíveis erros relacionados ao desconhecimento de espécies ou extrapolação de outras para regiões onde não se apresentam naturalmente.

Além disso, tomando como referência as fontes utilizadas na elaboração do modelo de orientação do conceito se observa no caso da obra de Yamamoto (2017), uma utilização da característica referente a presença de pelos como aspecto norteador na identificação de mamíferos, quando ao mesmo tempo, cita alguns mamíferos marinhos como exemplos de animais da classe em questão gerando uma contradição difícil de ser assimilada pelo estudante, sobretudo por se tratar de um material específico para os anos iniciais do ensino fundamental.

Assim, se cria um ambiente didático propício para que o aluno, ao invés de se orientar pelas propriedades e características invariantes, recorra muitas vezes a estratégia da memorização e da ideia do pensamento excludente quando se depara com um exemplo destoante da explicação do professor que nesse caso recorre a ideia da excepcionalidade como forma de burlar os limites de generalização impostos por uma orientação particularizada.

Este fato é explicado na TPFAMC pelo grau de generalização, ou seja, a medida que uma base orientadora é particular, seu limite de generalização e, consequentemente, sua aplicação em situações diversas de aprendizagem se torna bastante restrita o que exige uma estratégia de tentativa-erro por parte do aluno que acarreta em muitas possibilidades de erro e assim, um ambiente desmotivador de aprendizado (Nuñez \& Ramalho 2018; Mendonza \& Delgado 2020).

Por isso, como defendem Nuñez \& Ramalho (2018), planejar a atividade didática em função do EBOCA se configura como uma estratégia para minimizar essas situações de orientação restritiva e particularizada, pois a medida que o professor elabora uma orientação de referência capaz de abranger o maior número de situações-problema, reduz a necessidade de memorização por parte do aluno, uma vez que ao reconfigurar sua orientação em termos generalizados, se amplia a transferência de aprendizagem frente uma classe de situaçõesproblemas.

Portanto, o processo de aprendizagem não precisa ser fragmentado e interpretado como um amontoado de casos particulares que geram uma compreensão passível de inferência, ao contrário, o EBOCA estabelece um padrão de generalização de compreensão, o qual, baseado nas características essenciais a um dado fenômeno, objeto, ação ou conceito, aplica ao aprendizado, um nível de consciência sobre o conhecimento operativo para se chegar na assimilação do modelo do objeto de modo que o estudante passa a compreender a partir de um contexto lógico e não meramente reprodutor de instruções para se chegar a uma determinada resposta.

Isso se torna evidente no caso específico do estudo dos mamíferos, por exemplo, ao se constatar, conforme presente no material didático de autoria de Yamamoto (2017), quando correlaciona a presença de pelos e a amamentação como características exclusivas dos mamíferos e que se torna equivocada ao se confrontar com as pesquisas trazidas que expõe o caso da aranha da espécie Toxeus magnus, que alimenta seus filhotes com substância similar ao leite produzido pelos bovino (Dong et al. 2019).

Analisando essas evidências que contrastam informações tidas como específicas mas que na verdade não se configuram como aspectos invariantes, é que se chama a atenção para a importância didática de se pensar no EBOCA como um objetivo de aprendizagem nas aulas de ciências, pois, em consonância com o que defende Talízina (2000), essa orientação pautada no 
método teórico da atividade, garantem a representação do modelo estrutural-funcional que possibilita pensar em termos de uma constructo lógico acerca do conceito.

Consequentemente, em termos didáticos, assim como destacam os estudos acerca da formação de habilidades através do sistema orientador da ação e de sua etapa materializada (Pereira \& Nuñez 2013; Nuñez \& Ramalho 2018), a compreensão do EBOCA por parte dos estudantes, a medida que assimilação ocorre, permite uma regulação do processo de forma que a aprendizagem ocorre sem um rigor sequencial das operações.

Portanto, em situações que o estudante se depara com contextos diferentes, ele precisa pensar na orientação que utiliza para responder as tarefas, podendo inclusive reorganizar ou mesmo modificar seu EBOCA. A esse processo é o que Galperin (1986) considera como as mudanças qualitativas sobre um conhecimento já internalizado. Assim, em relação ao EBOCA proposto para este estudo, o qual tem como função primordial servir de representação para a aprendizagem e ensino de mamíferos em função de sua máxima generalização e estabilidade da aprendizagem, após a determinação da invariante se elabora a caracterização demostrada no Quadro 4, a seguir:

Quadro 4. Caracterização do EBOCA para ensinar o conteúdo de mamíferos.

\begin{tabular}{lll} 
Modelo do Objeto & \multicolumn{1}{c}{$\begin{array}{c}\text { Modelo da ação/Operações } \\
\text { (O que é um mamífero?) }\end{array}$} \\
\hline Ser vivo de reprodução sexuada, com fecundação e e identifica um mamífero?)
\end{tabular}

Um dos diferenciais contidos na elaboração desse esquema orientador está no fato de ao representar os conhecimentos operativos, privilegia, ao contrário do que a escola faz, a assimilação do conhecimento procedimental em relação a um conceito, o que, de acordo com Talízina (2000), concilia os aspectos estruturais para o desenvolvimento cognitivo dos estudantes, pois associa ao conceito, suas invariantes operativas responsáveis pela formação das ações conscientes, que à medida que se automatizam, se transformam em hábitos.

No caso específico do conceito de mamíferos, diferente das bases orientadoras que originaram a caracterização da invariante (Quadro 2), as quais não relacionam as categorias conceituais e procedimentais em uma só orientação, esse EBOCA dispõe de uma referência para a aprendizagem em uma perspectiva sistêmica quanto ao aspecto estrutural e funcional.

De acordo com a TFPAMC, essa natureza possibilita ao sujeito uma maior independência para resolver problemas e ao mesmo tempo contribui para o desenvolvimento da consciência na execução da atividade (Fariñas 2010). Corrobora, portanto, para responder à questão inicial desse estudo no sentido de garantir uma via didático-metodológica para sistematizar o ensino de mamíferos de acordo com uma concepção cognoscitiva do desenvolvimento, isto é, em função de uma mediação que promova a aquisição de sentidos em torno do significado científico.

Do ponto de vista das áreas temáticas preconizadas pela BNCC, alia as questões morfológicas, fisiológicas e estruturais relacionadas às espécies associadas à classe de mamíferos, nesse sentido, possibilita ao estudante compreender o conceito em uma dimensão explicativa, que na perspectiva histórico-cultural se associa à formação e desenvolvimento das funções psíquicas do aprendizado.

Conforme esclarece Yarochevsky \& Vygotski (1989), essas funções psicológicas superiores passam pela mediação semiótica, a qual, em função da formação de conceitos 
científicos estabelece a relação entre o signo e a linguagem, promovendo assim $o$ desenvolvimento da consciência em função de suas representações mentais.

Portanto, no âmbito desse estudo, o EBOCA adquire essa dimensão semiótica, a qual, a partir de seu plano materializado possibilita a assimilação conceitual que, por sua vez, tem potencial simbólico para intercambiar o significado externo ao plano das representações mentais ou internas, se configurando assim como uma evidência do desenvolvimento cognoscitivo.

\section{Considerações finais}

Um estudo dessa natureza evidencia a possibilidade de, a partir da caracterização da invariante conceitual, se estabelecer uma prerrogativa de ensino que contribua para a assimilação de conceitos científicos em função de um caráter estável de aprendizagem, à medida que a internalização passa a considerar os aspectos da generalização em detrimento de situações particulares.

Apesar de retratar um recorte do ensino das ciências para o Ensino Fundamental, a partir do conceito de mamíferos, dada sua descrição metodológica por meio do método da Atividade, demonstra uma viabilidade na prática docente dos professores envolvidos com o ensino das ciências, pois, à medida que o professor adquire a consciência de que a orientação da ação é um sistema estruturante da aprendizagem, pode exercer a atividade em sala de aula de forma mais efetiva em consonância com as prerrogativas necessárias à alfabetização científica.

Dialogando com estudos de natureza histórico-cultural acerca do processo de formação de ações mentais e habilidades cognitivas, as investigações sob a perspectiva do psiquismo e da teoria da atividade, sobretudo em função da TFPAMC, evidenciam que investir em propostas de natureza didática que favoreçam a formação do pensamento teórico auxilia na compreensão dos estudantes.

Além disso, em relação ao ensino dos conceitos demonstra ser uma via para se pensar tanto o processo de ensino-aprendizagem, como o contexto da formação desses professores, pois, a medida que se tem uma representação do modelo do objeto e da ação relativas ao EBOCA, é possível se elaborar sistemas didáticos compatíveis com as necessidades individuais dos alunos em função de suas dificuldades e potenciais.

Por essas razões se acredita que a TFPAMC, e as aplicações das ideias de P. Ya. Galperin no contexto do ensino das ciências possibilita um ganho qualitativo no processo de assimilação dos conceitos e, consequentemente, na aprendizagem em sala de aula. Essa pesquisa possibilita aos educadores, estudantes das licenciaturas e comunidade científica em geral um recorte dos trabalhos de pesquisa aplicados no âmbito da escola pública em ações que ocorrem sob a parceria da Universidade e que podem servir como um ponto de partida para discussões acerca da didática das ciências e o processo de ensino-aprendizagem.

\section{Referências}

BRASIL S. (2017) Base Nacional Curricular Comum-BNCC. 600 p.

Davidov V. \& Shuare M. (1988) La enseñanza escolar y el desarrolo psiquico: investigación psicológica teórica experimental. Moscu: Progresso. $222 \mathrm{p}$.

Dong B., Quan R. \& Chen Z. (2019) Prolonged milk provisioning and extended maternal care in the milking spider Toxeus magnus: biological implications and questions unresolved. Zoological Research, 40(4): 241-243. https://doi.org/10.24272/j.issn.2095-8137.2019.041

Fariñas L.G. (2010) Axiología y epistemología del enfoque histórico cultural: para uma praxis humanista. Disponível em: http://galatea.uacm.edu.mx (Acessado em 30/09/2020).

Galperin P.Ya. (1982) Intellectual capabilities among older preschool children: on the problem of training and mental development. Chicago: University of Chicago. $526 \mathrm{p}$.

Galperin P.Ya. (1986) Sobre el método de formación por etapas de las acciones intelectuales en antología de la psicología pedagógica y las edades. La Habana: Editorial Pueblo y Educación. $346 \mathrm{p}$. 
Galperin P.Ya. (2001) Sobre la formación de los conceptos y de las acciones mentales: la ciencia psicológica en la URSS (p. 45-56). In: Rojas L.Q (Comp.). La formación de las funciones psicológicas durante el desarrollo del niño. Tlaxcala: Universidad Autónoma de Tlaxcala.

Galperin P.Ya, Zaporózhets A. \& Elkonin D. (1987) Los problemas de la formación de conocimientos y capacidades en los escolares y los nuevos métodos de enseñanza en la escuela (p. 306-316). In: Shuare M. (Org.). La psicologia evolutiva y pedagógica en la URSS. Antología. Moscú: Editorial Progreso. 350 p.

Instituto Nacional de Estudos e Pesquisas Educacionais Anísio Teixeira - INEP (2015) Pisa. Disponível em: http://download.inep.gov.br (Acessado em 30/09/2020).

Longarezi A.M. \& Puentes R.V. (2017) Ensino desenvolvimental. Antologia. Uberlândia: EDUFU. $242 \mathrm{p}$.

Mendoza H.J.G. \& Delgado O.T. (2020) Proposta de um esquema da base orientadora completa da ação da atividade de situações problema discente. Obutchénie: Revista de Didática $e$ Psicologia Pedagógica, 4(1): 180-200. https://doi.org/10.14393/OBv4n1.a2020-56482

Nuñez I.B. (2009) Vygotsky, Leontiev, Galperin: formação de conceitos e princípios didáticos. Brasília: Liber livro. 100 p.

Nuñez I.B. \& Pacheco O.G. (2013) Formação de conceitos segundo a Teoria de Assimilação de Galperin. Cadernos de Pesquisa, 105: 92-109.

Nuñez I. \& Ramalho B. (2013) A formação de habilidades no contexto escolar: contribuições da teoria de P. Ya. Galperin. 34 ${ }^{\text {a }}$ Reunião Anual da Anped. Rio de Janeiro: Anped. 17 p.

Nuñez I.B. \& Ramalho B.L. (2018) Diagnóstico do nível de desenvolvimento da orientação de uma ação, em Química Geral, com futuros professores: contribuições da Teoria de P. Ya. Galperin. Obutchénie: Revista de Didática e Psicologia Pedagógica, 2(2): 412-439. https://doi.org/10.14393/OBv2n2a2018-6

OCDE (2013) PISA 2012 assessment and analytical framework: Mathematics, reading, science, problem solving and financial literacy. [S. 1.]: OECD Publishing, 2013. Disponível em: https://bit.ly/2XhQVpM (Acesso em: 20/05/2020).

OCDE (2015) Programa da OCDE Internacional de Alunos-PISA. Itens liberados de ciências. [S. l.: s. n.], 2015. Disponível em: https://bit.ly/2zfTHUU (Acesso em: 20/05/2020).

Oliveira M., Costa S.C.S. \& Costa S. (2013) A abordagem de mamíferos nos livros didáticos de ciências. $2^{\circ}$ Simpósio de Integração Científica e Tecnológica do Sul Catarinense: 767.

Pereira J. \& Nuñez I.B. (2013) Gráficos cartesianos nos livros didáticos de Química do PNLD 2012. Atas do IX Encontro Nacional de Pesquisa em Educação em Ciências. Águas de Lindóia, São Paulo. Problem Solving and Financial Literacy, OECD Publishing.

Puentes R.V. \& Longarezi A.M. (2017) A didática desenvolvimental: seu campo conceitual na tradição da psicologia histórico-cultural da atividade. Fundamentos psicológicos e didáticos do ensino desenvolvimental. Uberlândia: Edufu. 224 p.

Rosa M.D.A. (2017) O Uso do Livro Didático de Ciências na Educação Básica: Uma Revisão dos Trabalhos Publicados. Contexto \& Educação, 32(103): 55-86. https://doi.org/10.21527/21791309.2017.103.55-86

Salmina N.G. (1988) Análisis lógico-psicológico de los procedimientos para discutir la asignatura docente. La Habana: Educación Superior Contemporânea. 11 p.

Silva L.G.L., Santos C.F. \& Lima R.N. (2012) Uma análise crítica do conteúdo mammalia em livros didáticos do Ensino Médio utilizados em escolas públicas e privadas de Floriano-PI. VII Congresso Norte Nordeste de Pesquisa e Inovação. Tocantins: Editora IFTO.

Tacca M.C.V.R \& González F.L.R. (2008) Produção de Sentido Subjetivo: As Singularidades dos Alunos no Processo de Aprender. Psicologia: ciência e profissão, 28(1): 138-161.

Talízina N.F. (1987) Procedimientos iniciales del pensamiento lógico. Moscú: Editorial Progresso. $79 \mathrm{p}$.

Talízina N.F. (2000) Manual de Psicología Pedagógica. México: Universidad Autónoma de San Luís Potosí. 336 p.

Vygotski L.S. (1993) Obras escogidas II. Madrid: Visor. 595 p.

Yamamoto A.C.A. (2017) Buriti mais: Ciências - 1 Ano. São Paulo: Moderna. 
Base orientadora para o conteúdo de mamíferos

Yarochvsky M.G. \& Vygotski L. (1989) A procura de uma nova psicologia. Ênfase: 1-2. 